\title{
The Quantum Oscillatory Modulated Potential-Electric Field Wave Packets Produced by Electrons
}

\author{
Waldemar Wolney Filho \\ Instituto de Flsica, Universidade Federal de Goiás, Goiânia, Brasil \\ Email: wwolney@yahoo.com.br
}

Received 31 January 2015; accepted 23 November 2015; published 26 November 2015

Copyright (C) 2015 by author and Scientific Research Publishing Inc.

This work is licensed under the Creative Commons Attribution International License (CC BY). http://creativecommons.org/licenses/by/4.0/

(c) () Open Access

\section{Abstract}

In this work we are formulating a new theory for describing the waving nature of a microscopic electric particle. Based on the predictions of the Quantum Oscillatory Modulated Potential-QOMP, for describing the interaction between two microscopic electric particles, electron-electron, for instance, we are postulating that the waving behavior of these particles may be an attribute of the charges of the particles and not their masses as currently accepted. For a microscopic electric charge, we are presenting new arguments showing that the electric field in the vicinity of a microscopic charge is spatially waving and can be determined as the gradient per unit of charge of this new quantum interaction potential, with use of an appropriated phase factor to account for the behavior of an unbound electron. Differently of what is predicted by the classical Coulomb electric field, when a charged particle is moving under the action of a potential of $V$ volts, the new electric field existing around the charge has the form of a wave packet. For typical values of the potential $V$, the wavelength of the waving electric field is in very good agreement with those experimentally observed with diffraction of electrons in crystalline solids.

\section{Keywords}

Potential, Electron, Wavelength, Wave Packet, Bosonic Atom

\section{Introduction}

In this paper we are revisiting an old, but intriguing and challenging topic of the modern physics-the phenomenon of particle-wave duality. Even considering that the phenomenon has been investigated by many physicists, for more than a century, it seems to us that the subject still presents some obscure points and deserves fur- 
ther investigation.

In the development of this work, it was able to observe that the new potential, used to describe the interaction between two microscopic electric charges, two electrons for example, presents surprising but strong evidences for a possible formation of electron pairs in an environment of a dense cluster or a dense beam of electrons. Otherwise, observing this possibility from another point of views, we are conjecturing that the electron-electron interaction indicates a possible existence of a kind of a bosonic atom, an "atom" with characteristics similar to those of the hydrogen atom but with positive energy interaction. Another perspective to predict the existence of this new type of atom is the formation of a pair of electrons, such as the Cooper pair used in the BCS theory, in the superconductivity ${ }^{1}$.

In order to update the reader with the subject, without going into too much details, first we will make a brief overview of two important physical models, developed by eminent physicists of the last century. In the last years of the 20 decade, European physicists worked enthusiastically to give an explanation for this seemingly irreconcilable problem - the particle-wave behavior. In the particular case of the electron, one of the first attempts to explain this phenomenon was made by Schrödinger, de Broglie and Born [1]. In a double-slit experiment, with the use of a beam of electrons, these physicists tried to explain the phenomenon, associating a probabilistic wave to each electron of the beam, looking for an explanation to the observed interference patterns in crystalline solids. Here, as mentioned before, we are not going to entry in details of the method. But, in general way, the results of the method were in accordance with the experimental observations. What was not clear in their proposed physical model was the essence or, in other words, what was the real cause for the formation of the probabilistic waves associated to the electron. Adopting another point of view to solve the puzzle, years later a different approach was idealized and formulated by the American physicist Richard Feynman [1] [2]. Based on the results of experiments already available and considering that at the end of the 20 decade, the physicists have come to the conclusion that any attempt made to determine, simultaneously, the ambiguous characteristic of the electron, particle or wave, the interference of the observer in the process invalidates the experience. Later, in Section 4, we will return to discuss this point.

In a double-slit experiment, Feynman, trying to elucidate the problem, took a rather radical attitude. According to his point of view, there is no need to join the electron a wave of probability. He argued that the electron, leaving the source could pass simultaneously from either of the two slits of the apparatus, following an infinity number of possible paths until reaching the phosphorescent screen to produce the interference pattern. According to the view of Feynman, the electron "sniffs", simultaneously, all paths connecting the beginning to the end of the trip and choose the most convenient one. Based in this premise Feynman presented a new approach to the problem. According to the perspective of Feynman, there is no need to create a wave of probabilities for the electron. From his point of view, the electron, always seen as a particle, may reach a certain point on the screen, and is the result of the combined effect of all possible ways to get there. This method is known as "sum over trajectories" and it is one of the major contributions of Feynman for the development of quantum mechanics [1]. However, even from his point of view, the possibility of an electron to take simultaneously different paths, more than this, to take an infinite number of paths, constitutes a legitimate objection to our common sense. More than that, this seems absurd. Other prominent physicists, including Albert Einstein, never agreed with the probabilistic point of view of quantum mechanics. By the end of his life, Einstein was still believing that there was a different way, a non-probabilistic manner, to explain the quanta phenomena. On the other hand, the scientific community recognizes that in the microscopic world, the quantum mechanics presents a large number of surprises. The results of the calculations presented by Feynman, based on his method, agree with the results of the method previously mentioned. Also, it agrees with the experimental results. Thus, for the majority of the scientific community, this problem seems to be solved and nothing else remains to be done. However, this is not completely true. As already stated previously, some obscure points with respect to this subject, still persist and deserve a new approach in an attempt to find a more convincing explanation.

Related to the subject under discussion, at this point, it is quite logical to ask the following questions: What physical property of the electron is responsible for generating the, so called, wave of probability associated to this particle? Is it the mass of the electron? Is it the charge? Or is it the effect caused by an electric or a magnetic field associated to the particle? The electron has mass, has charge, has spin and, consequently, we like it or not, there is an electromagnetic field associated to it. In this paper we are trying to find an answer to these questions, adopting a quite different point of view.

${ }^{1}$ This subject will be investigated with details in the fifth part of this series of work. 
Nowadays, one of the theoretical models more acceptable to explain the dual behavior of a microscopic particle, such as the behavior of an electron in the double-slit experiment, is to use the principle of superposition of an infinite number of plane waves, to built up a wave packet around the particle. Simple plane waves can be obtained from the solution of the Schrödinger's wave equation for a free particle. However, we must observe that the only property of a free uncharged particle is its mass. Thus, the energy operator in the Schrödinger's wave equation, only involves the kinetic part of the Hamiltonian operator. So, for a free particle moving in space, the Schrödinger wave equation takes the form

$$
-\frac{\hbar^{2}}{2 m} \nabla^{2} \Psi(\boldsymbol{r}, t)=i \frac{\partial}{\partial t} \Psi(\boldsymbol{r}, t)
$$

It solution is a simple plane wave, written as

$$
\Psi(\boldsymbol{r}, t)=A \mathrm{e}^{i(\boldsymbol{k} \cdot \boldsymbol{r}-\omega t)}
$$

where $A$ is an arbitrary constant. However, a plane wave like this, occupies the entire space. Thus, it becomes impossible to determine the position of the particle, whose behavior is described by this wave function, because the particle can be anywhere in the space. So, to overcome the difficulty to localize particle, the physicists, artificially, created a wave packet around the particle. This wave packet intends to confine the particle inside a small region of space. The formation of a wave packet can be accomplished by making the superposition of a large number or, better yet, an infinite number of plane waves around a specified momentum $\boldsymbol{p}_{o}=\hbar \boldsymbol{k}_{o}$ of the particle. Thus, using this procedure to built up a wave packet, the only physical property of the particle that takes part in the process is the mass of the particle. In this case, the only force field that can be associated to the particle in movement is the gravitational field. However, for a small mass, comparable to the mass of the electron, which is approximately $10^{-30} \mathrm{~kg}$, the gravitational field associated to this particle is completely negligible and can be discarded. Therefore, what is or what are other properties of the particle that can be considered for the formation of a wave packet? Let's try to answer this question in the next paragraphs.

In actual experiments of electrons diffraction, the kinetic energy of a beam of electrons is dependent on both, the mass and the charge of the particle, and consequently, on the electric field existing in its neighborhood. Regardless of the considerations or conjectures taken attempting to explain the dual behavior of a charged particle like electron, the intrinsic electric field in the neighborhood of the particle cannot be disregarded. In principle, according to the Coulomb potential, the formation of a narrow and dense beam of electrons should be divergent due to the repulsive electron-electron interaction. In real experiments using a thin and dense beam of electrons, the electric force between these particles must be repulsive and, in principle, the electron beam should be divergent. However this is not observed or, the divergence effect is very small and can be neglected

Experiments with beams of electrons (charged particles) and X-rays (waves) scattered by thin metallic foils show diffraction patterns with great similarities. The similarity of the diffraction patterns shows that electrons can behave like X-rays, displaying wave-like properties. In 1924, the French Physicist Louis de Broglie postulated that associated to the particle there is a wave packet, given to it wave-like properties. From the special theory of relativity, Einstein have shown that the wavelength associated to a photon can be written as [3]

$$
\lambda=\frac{h}{p},
$$

where $h$ is the Planck constant and $p$ is the momentum of the photon. The waving nature of the photon or its particle-like behavior appears when an electron in an atom changes its quantum energy state from $E_{i}$ to $E_{j}$, with $E_{j}>E_{i}$. When this quantum transition occurs, the electron in the atom drops from a quantum state of higher energy to a quantum state of lower energy, emitting the excess of energy in form of electromagnetic radiation, a photon - a tiny electromagnetic wave packet. Since for a bound electron, both energies $E_{i}$ and $E_{j}$ are negatives, $E_{i}$ is less negative than $E_{j}$, a necessary condition for a photon emission. Let us attribute the relativistic linear momentum of the emitted photon to the variation of the relativistic quantized linear momentum $p_{i j}$ of the electron in the atom. This variation of the linear momentum of the electron may be written in the form,

$$
p_{i j}=m v_{i j}=\frac{m_{m}}{\left(1-\frac{v_{i j}^{2}}{c^{2}}\right)^{\frac{1}{2}}} v_{i j},
$$


where we are considering $m_{m}$ as the minimum relativistic mass of the electron. In the Equation (4), the minimum relativistic mass takes place when $v_{i j} \rightarrow 0$, what makes $m \rightarrow m_{m}$. In an atom this electron transition occurs between two quanta states with large quantum numbers $n_{i}$ and $n_{j}$. From the above equation we can observe that the relativistic mass of the electron, when occupying a particular energy quantum state $E_{n}$ of the atom, may be written as

$$
m_{n}=\frac{m_{m}}{\left(1-\frac{v_{n}^{2}}{c^{2}}\right)^{\frac{1}{2}}},
$$

which increases when the velocity $v_{n}$ of the electron increases. On the other hand, it is known that, in an atom, the velocity $v_{n}$ of the electron, for a specified energy quantum state $E_{n}$, may be expressed by [4].

$$
v_{n}=\frac{1}{4 \pi \epsilon_{o}} \frac{Z e^{2}}{n \hbar}
$$

where $Z$ is the atomic number. This equation indicates that the largest velocity of the electron occurs when it is occupying the ground state of the atom, $n=1$. The Equations (5) and (6) indicate that for a bound electron, the relativistic mass of the particle decreases when the quantum number $n$ increases. Then, the smallest value of the mass of the electron occurs when $n \rightarrow \infty$. That is, when the electron becomes free and the relativistic mass $m$ becomes equal to its minimum value, $m=m_{m}$. The reader must observes that in the Equation (4), we are not using the concept of rest mass for the electron because, according to the Heinsenberg uncertainty principle, $\Delta x \Delta p=\hbar$, for microscopic particle such as the mass of electron, strictly, this particle never will be at rest in the laboratory frame of reference-LFR. Being so, rigorously, the rest mass for a microscopic particle like the electron, does not exist. However, for what is called a nonrelativistic electron, that is the case when its velocity $v_{i j} \ll c$, we can make the approximation $m_{m} \simeq m_{0}$, what is called rest mass of the electron. Under this condition, the variation of the linear momentum of the electron, when the atom changes the quantum state from $E_{i}$ to $E_{j}$, can be written in the form

$$
p_{i j}=m_{o} v_{i j}=\frac{E_{i}-E_{j}}{c} .
$$

But, in a more general way, taking into account transitions occurring in heavy atoms, where $v_{i j}$ may be considered large, this equation may be written in a slightly different form

$$
m v_{i j}=\frac{h f_{i j}}{c}=\left(\frac{h}{c^{2} \tau_{i j}}\right) c,
$$

where $m$ is the relativistic mass of the electron and, $\frac{h}{c^{2} \tau_{i j}}=\frac{E_{i j}}{c^{2}}$ being the relativistic mass of the photon, $\tau_{i j}$ is the transit time for the electron to move between two quanta states of the atom. During this time interval, the atom is loosing energy, a photon is being created and gaining the relativistic quantum energy $\left|E_{i j}\right|=\left|E_{i}-E_{j}\right|=h f_{i j}$.

As it can be seen, the quantum relativistic Equation (8), has a simple but a profound physical significance. It informs us, in a quite clear manner, the nature of the dual character of the particle. The equation shows that the dual behavior of a charged particle, the electron for instance, is a pure electromagnetic phenomenon associated to the particle. This can be seen in the following way; the phenomenon of wave particle duality is due to an electric field associated to the charge and a magnetic field associated to the spin of the particle, in this case, the spin of the electron. In the Equation (8) we can observe that the left side of the equation is a product of the relativistic mass of the electron times the transit velocity $v_{i j}$ when the electron in the atom jumps back from an excited energy state $E_{i}$ to a lower energy state $E_{j}$.

The arguments presented above, show that the electron carries around it an electromagnetic field that changes its interaction configuration with the nucleus of the atom during the time of flight of the particle, or equivalently, during the time the electron is tunneling through the potential barriers as predicted by the new potential, the Quantum Oscillatory Modulated Potential QOMP [5] [6], which is given by the formula 


$$
U(r)=-\frac{1}{4 \pi \epsilon_{0}} \frac{e^{2}}{r} \cos ^{2}\left(\xi_{n} r\right)
$$

where $\xi$ is a space frequency, it is a quantized parameter, given by

$$
\xi_{n}=\left(\frac{1}{4 \pi \in_{0}}\right) \frac{\pi \mu e^{2}}{n^{2} \hbar^{2}}
$$

where $\mu$ is the reduced mass of the system proton-electron in the hydrogen atom, which is

$$
\frac{1}{\mu}=\frac{1}{m_{p}}+\frac{1}{m_{e}}
$$

For the hydrogen atom, according to the potential predicted by Equation (9), the changes of the interaction configuration is shown in Figure 1, when the electron in the atom changes its energy from $E_{2}$ to $E_{1}$. The right side of the Equation (8) is the product of the relativistic mass of the photon and its velocity $c$. Then, by a question of symmetry or conciliation, both sides of the equation must present, simultaneously, both aspects, relativistic mass and wave. From a purely mathematical point of view, the Equation (8) reflects the particle-wave dual behavior for the electron.

According to what we have seen previously, we have a clear indication that associated to a bound electron there is a quantum electromagnetic field and, associated to a free electron, we are postulating the existence of an electromagnetic field in the form of a wave packet, moving with the velocity particle. Because of this we can, appropriately, say that the electron is an electromagnetic-particle-wave manifestation of nature, since the electron carries, simultaneously, both aspects, particle and wave. As we will see in the next paragraph, this manifestation will be identified as the agent responsible for the observed particle-wave duality as it is observed in experiments with diffraction of electrons in solids.

The arguments presented above may be extended to other atomic particles, like the proton and others known charged particles. However, we must observe that the neutron, an uncharged particle, also presents similar effect and is frequently used in experiments of neutrons diffraction. Being so, how this argument could be accepted for explaining the neutrons diffraction, being these uncharged particles? We believe that the answer to this question is the fact that the neutron, as well as the hydrogen atom, that also present similar effect, are composite particles. The neutron, as proposed by Gell-Mann and G. Zweig [7] is built up of three kinds quarks, one labeled up with charge $+2 / 3$ (u) and two others labeled down with charge $-1 / 3$ (d) and $-1 / 3$ (d). Then, another way to represent the neutron is (udd). These particles must have associated to them an electric dipole moment-the so-called nEDM. In this case, they will present similar electromagnetic effects, like the electrons. Currently there are at least four experiments trying to measure the $\mathrm{nEDM}$ [8]. Theoretical typical predictions for the neutron electric dipole moment range between $10^{-27} \mathrm{Cm}$ and $10^{-30} \mathrm{Cm}$ [9]. For the hydrogen atom, the QOMP mentioned before, predicts that the electron, when occupying the fundamental state of the atom, is separated from the proton with an average radial distance $+2 / 3\left\langle r_{1}\right\rangle=0.529 \times 10^{-10} \mathrm{~m}$ [5] [6], what predicts the existence of an average electric dipole moment $\langle p\rangle=0.847 \times 10^{-29} \mathrm{~cm}$ for the hydrogen atom. The configuration of this atom is shown in Figure 1. The existence of this electric dipole moment is not revealed when the Coulomb potential is used to describe the interaction proton-electron of the atom. The existence of the hydrogen atom electric dipole moment ( $h E D M)$, may constitutes a great break-through to explain the formation or even the existence of the $\mathrm{H}_{2}$ molecule. This idea will be explored further elsewhere ${ }^{2}$.

According to what we have seen previously, it is quite clear that, exists associated to the particle an electromagnetic field around it. Because of this, the waving behavior of the particle must be an attribute of its charge, creating around them waving electric fields. Thus, for the microscopic particles, like the electron, the proton and others known particles, a better term to describe them would be "particle-waves" because, in fact, they are electromagnetic wave particles, since, both aspects are inherent to these atomic physical objects. At this point, an interesting question is: What is the form of the electric field associated to a free electron in movement? This question will be answered in the next sections.

In what follows, for sake of simplicity, we will consider the electron as a spinless particle. With this

\footnotetext{
${ }^{2}$ At present, we are investigating the possibility that an interaction electric dipole-dipole can explain in a very simple way the formation of the hydrogen molecule.
} 


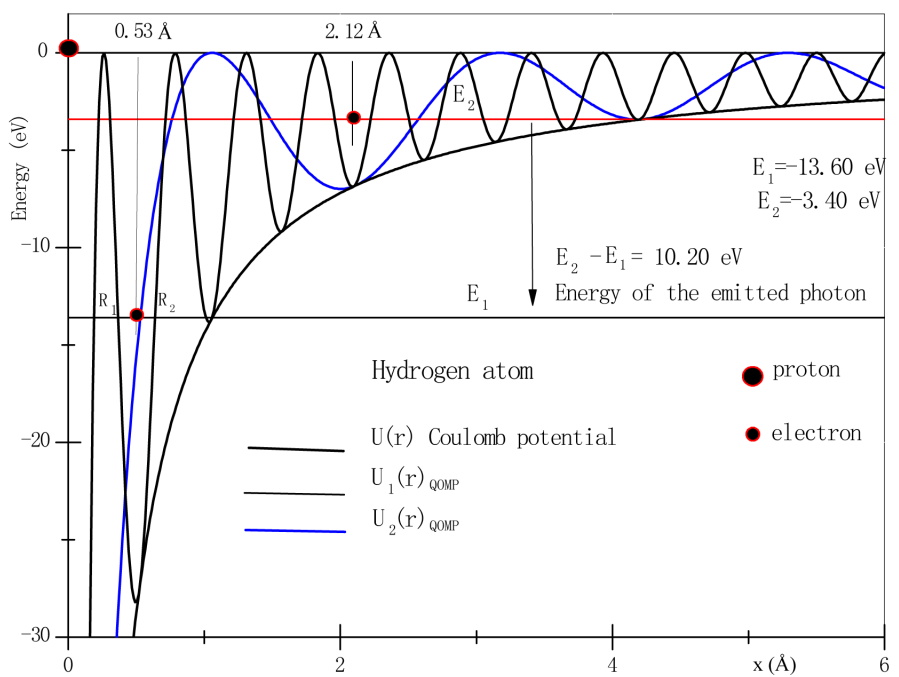

\begin{abstract}
Figure 1. Change of the proton-electron energy configuration in the hydrogen atom for the electron occupying the ground state of the atom $n=1,\left\langle r_{1}\right\rangle=0.53 \AA, E_{1}=-13.60 \mathrm{eV}$, the black line. And $n=2,\left\langle r_{2}\right\rangle=2.12 \AA, E_{2}=-3.40 \mathrm{eV}$, the red line. Also is shown the energy of the quantum transition $E_{2}-E_{1}$.
\end{abstract}

consideration, the magnetic part of the electromagnetic field, that due to the spin of the electron, will not be considered for the determination of the form of the wave packet we are looking for. Here, our concern will be solely directed to find the form and the characteristics of the electric field associated to the particle.

As we have seen previously, a photon is generated when the electron in an atom makes a quantum transition. In this work we are arguing that transitions occur when the electrons are tunneling through finite potential barriers as created by the new interaction potential, the QOMP, previously described. Differently of the classical Coulomb potential, when this new potential is used to describe the interaction proton electron in the hydrogen atom, the electron is not orbiting the nucleus of the atom, but moving back and forth oscillating radially between two classical turning points $R_{1}$ and $R_{2}$, like a quantum harmonic oscillator. See Figure 1 . Then, even in the absence of any other field, there is a fluctuation of the electric field inside the quantum wells of the atom, with the electron, eventually, falling to a lower energy state by tunneling effect, causing a photon emission by the atom, as previously described. Of course, the inverse of this process is the photon absorption by the atom.

In 1923, the same Louis de Broglie postulated the existence of another important relation in which, the linear momentum $p$ of the particle is also related to the space frequency $k$ by

$$
p=\hbar k \text {, with } k=\frac{2 \pi}{\lambda} .
$$

Moreover, it is also known that a nonrelativistic charged particle with mass $m$, moving with velocity $v$, has kinetic energy $E_{k}$, given by

$$
E_{k}=\frac{p^{2}}{2 m} \Rightarrow p=\left(2 m E_{k}\right)^{\frac{1}{2}} .
$$

From Equations (12) and (13) we have the well known formula

$$
\lambda=\frac{h}{\left(2 m E_{k}\right)^{\frac{1}{2}}} .
$$

In particular, charged particles like protons or electrons, accelerated through a potential of $V_{o}$ volts, have kinetic energy equal to $E_{k}=e V_{o}$. The wavelength associated to these particles, is predicted by

$$
\lambda=\frac{h}{\left(2 m e V_{o}\right)^{\frac{1}{2}}} \text {. }
$$


This equation tell us that the de Broglie wavelength, besides its dependence upon the mass of the particle, it is also dependent on its charge and the accelerating potential $V_{o}$, which is an external agent acting on the particle.

\section{An Electric Field Wave Packet}

As explained previously, the Equation (3) predicts the wavelength associated to a photon, an electromagnetic wave packet. The Equation (15) predicts the wavelength associated to an electric particle, the electron for instance, accelerated through a potential of $V_{0}$ volts. A beam of X-rays and a beam of electrons presents similar effects when used to produce diffraction in crystalline solids. An X-ray photon can be seen as an electromagnetic wave packet, moving with the velocity of light $c$. In this work, we are postulating that the free electron also must be seen as an electromagnetic wave packet moving with the velocity of the particle. For this arguments to become more consistent and realistic it is necessary that the electric field associated to the particle in movement be spatially waving, forming a wave packet with size and wavelength appropriated to be diffracted as it is experimentally observed. An electric field with these characteristics may be formulated taking the gradient per unit of charge of the new interaction potential between two electrons. Similar to the Equation (9), the new potential may be written as

$$
U(r)=\frac{1}{4 \pi \epsilon_{0}} \frac{e^{2}}{r} \cos ^{2}\left(\chi_{n} r\right)
$$

where $\chi$ is the space frequency. Also, similarly to the interaction between the proton and the electron in the hydrogen atom, $\chi_{n}$ in the Equation (16) is a quantized parameter expressed by

$$
\chi_{n}=\frac{1}{4 \pi \in_{0}} \frac{\pi \mu e^{2}}{n^{2} \hbar^{2}}
$$

with $\mu$ being the reduced mass of the electron-electron interacting system. Here it is represented by

$$
\frac{1}{\mu}=\frac{2}{m_{o}}
$$

which, for a nonrelativistic condition, the reduced mass $\mu$ is one-half of the electron rest mass $m_{o}$. At this point we will open a parentheses in our presentation to make some comments about the electron-electron interaction potential as expressed by Equation (16). This potential presents an unexpected and a quite interesting interpretation. First, as it can be seen in Figure 2, the potential is not always repulsive as predicted by the classical Coulomb potential, also displayed in the figure. The new potential is null at every points where the quantization relation $\chi_{n} r=n \pi$ is satisfied, with $n$ being an integer number, different from zero. It is easy to see that this quantization relation is a direct consequence of the Heisenberg uncertainty principle. The new potential presents positive decreasing barriers with the $1 / r$ dependence. Seen this potential in another way; it presents barriers heights that decrease modulated by the classical Coulomb potential as shown in the same figure.

A quite interesting aspect of this potential is that it predicts the existence of kind of electron-electron bosonic particle. A kind of "atom" formed of two interacting electrons. That is an atom formed of two fermionic particles of spin $1 / 2$, producing an atom with spin $S=1$ (the two electron spins paired) or spin $S=0$ (the two spins unpaired). Because of this attribute we are calling it an electron-electron bosonic atom.

Figure 2 also shows the first three configurations for the electron-electron interaction and the four first energy levels of this new kind of atom. As mentioned previously, another way to see this kind of atom, is the possible formation of a kind of an electron pair, similar to the existence of Cooper pairs in the state of super-conductivity for some metals and metals alloys at very low temperatures, temperatures below $T_{c}$ [10] [11] $]^{3}$. In Section 4, we will see how this new kind of atom can affect the diffraction patterns in solids. Having called the attention of the reader for these particularities of the electron-electron interaction, let us return to our initial presentation, since this interesting subject will be investigated elsewhere, with great details.

Focalizing our attention just upon one electron of a beam, as previously explained, we can admit the existence of an electric wave packet moving with the same velocity of the electrons of the beam. However, for this arguments to become more realistic, it is necessary that the electric field associated to the particle in movement be

\footnotetext{
${ }^{3}$ This interesting subject will be detailed discussed in the fifth paper of this series.
} 


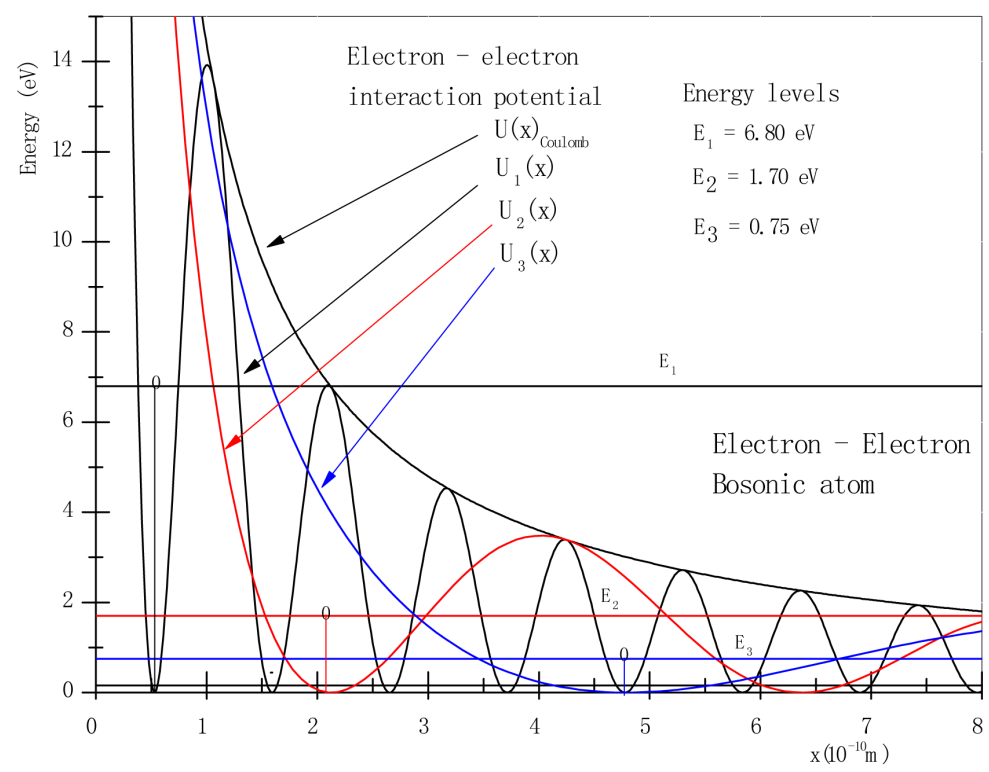

Figure 2. The electron-electron binding energy as predicted by the QOMP. The red horizontal line represents the binding energy for the ground state of the new atom, $E_{1}$ $=6.80 \mathrm{eV}$. The energies of the first three excited states of the new atom are also shown in the figure. The spin of the atom may be $S=1$, spins parallel, $S=0$ spins antiparallel. In the ground state of the atom, the electron is squeezed between the classical turning points $P_{1}$.

spatially waving, with a wavelength value appropriated to be diffracted as it is experimentally observed in the crystalline solids. An electric field with these characteristics may be formulated taking the definition of the electric field as

$$
\boldsymbol{E}(\boldsymbol{r})=-\lim _{q \rightarrow e} \frac{\nabla U(r)}{q} .
$$

The reason to use the limit $\left(\lim _{q \rightarrow e}\right)$ in the definition of the electric field, is because the electronic charge $e$ is the smallest electric charge existing in nature. Then, from Equation (16) we obtain the expression

$$
\boldsymbol{E}(r)=-\frac{e}{4 \pi \epsilon_{o}}\left[\frac{1}{r^{2}} \cos ^{2}(\zeta r)+\frac{\zeta}{r} \sin (2 \zeta r)\right] \boldsymbol{u}_{r},
$$

where $\zeta$ is the space-frequency associated to a free electron, which, due to the unusual periodicity of the new electric field, it may be written as

$$
\zeta=\frac{\pi}{h}(2 m e V)^{\frac{1}{2}}
$$

and $\boldsymbol{u}_{r}$ in the Equation (20), is a unit vector taken along the radial direction.

Currently, it is accepted that the electric field existing at a distance $r$ of a negative electric charge, an electron for example, is predicted by the well known classical Coulomb formula

$$
E(r)=-\frac{e}{4 \pi \epsilon_{o}} \frac{1}{r^{2}} \text {. }
$$

This classical electric field, near the electron, may be obtained from Equation (20), taking $\zeta=0$, what makes the linear momentum $p_{r}=\hbar \zeta$ of the electron to be null. As stated before, this makes the electron to be at rest in the LFR. This condition contradicts our initial supposition that the electron was moving with a velocity $v$, having kinetic energy $E_{k}=e V_{o}$. Besides, as explained previously, this condition also contradicts the Heisenberg uncertainty principle $\Delta r \Delta p_{r} \geq \hbar$. Consequently, the classical Coulomb electric field, as formulated by 
Equation (22), is not appropriate to represent the electric field in the vicinity of a microscopic electric particle. The same occurs with the classical Coulomb potential that is not suitable for describing the energy interaction between two microscopic electric charges [6]. This is a rather surprising revelation, since this interaction potential has been used in physics and chemistry for more than a century. On the other hand, as we will see below, the waving electric field, as formulated by Equation (20), does not present such, let's say, inconvenience.

The electric field wave packet as predicted by Equation (20) is quite different of the wave packets obtained with a superposition of an infinite number of plane waves, as briefly discussed previously and, with details, in the physics literature [3] [4] [12]. The wave packets obtained with the use of a superposition of plane waves for a free uncharged particle, fails to explain the origin of the waving aspect of the particle and, if the particle is charged like the electron, it does not show the $1 / r^{2}$ dependence, which is a characteristic of the electric field existing around the charge. In the next section we will discuss the use of this new electric field wave packet, using a beam of electrons, expecting to give an answer to this and other questions concerned to this subject.

\section{The Form of the New Electric Field in One Dimension}

For sake of simplicity, let us consider the case of an electric field produced by a nonrelativistic electron, moving through a potential of $V_{0}$ volts, in the $x$-direction. In this particular case the new electric field takes the form

$$
E(x)=-\frac{e}{4 \pi \epsilon_{o}}\left[\frac{1}{x^{2}} \cos ^{2}(\zeta x)+\frac{\zeta}{x} \sin (2 \zeta x)\right] .
$$

Considering negative and positive values of $x$, this electric field has the form of an one-dimensional wave packet with the charge, the electron, occupying the center of the packet. We are naming this new electric field by Electric Field Wave Packet_EFWP. Nonrelativistically, this electric wave packet moves with the velocity of the particle,

$$
v=\left(\frac{2 e V_{0}}{m}\right)^{\frac{1}{2}} .
$$

As a typical example for the prediction of the new electric field, let us consider the case of an electron, that has been accelerated through a potential $V_{o}=100$ volts. Thus, according to Equations (21) and (23), the electric field associated to this particle is given by the particular expression

$$
E(x)=-A\left(\frac{\cos ^{2}(1.88 x)}{x^{2}}+\frac{1.88}{x} \sin (2 \times 1.88 x)\right)
$$

where $A=14.40 \times 10^{-10} \mathrm{~N} / \mathrm{C}$. Figure 3 shows that this new electric field has the form of a wave packet as previously described. The de Broglie wavelength measured in the graphic is $\lambda=1.23 \AA$, which is in very good agreement with the value as predicted by Equation (15), $\lambda=1.22 \AA$. As shown in the same Figure 3, the effective size width of the electric wave packet is of the order of $10 \AA$, what is about the same order of magnitude of the inter-atomic space in solid crystals.

What seems odd about the shape of the wave packet shown in Figure 3, is the fact that, mathematically, the intensity of the electric field predicted by Equation (25) has an infinite value at the origin, the center of the packet. A particle with zero dimension is only a mathematical idealization Unlike this, the wave packet formed by the superposition of an infinite number of plane waves, has a finite value at this position, meaning that the density of probability $\Psi^{*}(\boldsymbol{r}, t) \Psi(r, t)$ to find the particle at the center of the wave packet is higher than it is elsewhere [5] [12]. Does this constitutes a failure to the newly created model? Our answer to this question is no. In what follows, we intend to answer this question making some considerations trying to show why this response is negative. On the contrary, instead of being a problem to be solved, this apparent contradiction, will help us to better understand the phenomenon of particle-wave duality.

Firstly we need to observe that Equation (20), as it is formulated, it describes the electric field of a point charged particle with zero space dimension. This is not the case for the electron, this particle has a finite size and, considered as a particle, its diameter is equal to the Compton wavelength. Thus, if we consider the electron as solid sphere with a uniform charge density, we can use the Gauss law to estimate the value of the electric field existing in its surface. With the use of Equation (21) we can calculate the phase factor $\zeta r$ of the oscillatory 


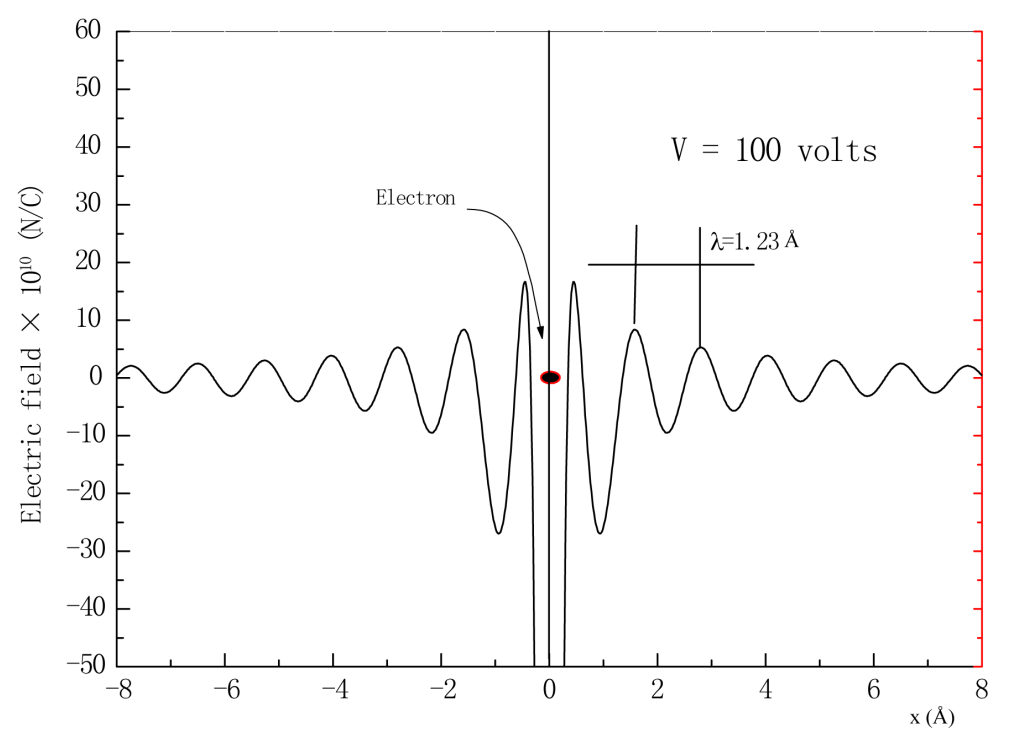

\begin{abstract}
Figure 3. The waving electric field-WEFP $E(x)$ for an electron that has been accelerated through $V_{o}=100$ volts. The measured wavelength is $\lambda=1.23 \AA$. The predicted value of de Broglie wavelength is $\lambda=1.22 \AA$.
\end{abstract}

terms in Equation (20). Taking the radius of the electron as $R=1.93 \times 10^{-13} \mathrm{~m}$ (half of Compton wavelength) and performing the calculation we find $\zeta R=0.0049$ radians. This small value makes $\cos ^{2}(\zeta R) \simeq 1$ and $\sin (2 \zeta R) \simeq 0$. Thus, according to Equation (20), the electric field on the electron surface, or even very closed to it, ceases to be oscillating, becoming equal to the classical electric field, with the $1 / r^{2}$ dependence.

With the information obtained above, we can use the Gauss law to calculate the electric field inside and on the surface of the electron, as stated previously, considered as a solid sphere of radius $R$. Accordingly, this electric field is predicted by the equation [7]

$$
E(r<R)=\frac{1}{4 \pi \in_{0}} \frac{e r}{R^{3}} .
$$

This shows that the electric field in the center of the electron, $r=0$ is zero. The value of the electric field at the surface of the particle is determined taking $r=R$. Its value is $E(r=R)=3.87 \times 10^{16} \mathrm{~N} / \mathrm{C}$. This represents a very large but, not an infinite value for electric field at the position of the electron.

In the graph shown in Figure 3, we can see that the value of the electric field at the position of the second most intense peak is about $500 \mathrm{~N} / \mathrm{C}$. This electric field is approximately $8 \times 10^{13}$ times smaller than the electric field near the surface of the electron. This information tells us that in order to obtain a pattern of electron diffraction in a double-slit experiment, it is necessary that the number of electrons in the beam be of the order of trillions. A small number of electrons in the beam will produce only a random distribution of small spots on the surface of the film, formed by the central peaks of the electric field. Thus, we can say that the discontinuities of the electric field as predicted by the Equation (25) and shown in Figure 3, becomes interrupted by the presence of the electron at the center of the wave packet. Therefore, we can conclude that the two physical models, in this particular aspect, are in agreement. Otherwise, as it will be discussed in Section 4, the great difference between the intensities of the central and the lateral peaks, is the crucial aspect to be observed for the comprehension of the dual behavior of the electron.

\title{
4. Predictions of the EFWP and Experimental Results
}

The correct prediction of the new electric field wave packet is in agreement with the results of many experiments reported in physics literature [3] [4]. The first successful experiment with a beam of elctrons incident on a crystal was produced in 1927 by Davisson and Germer. In this experiment was used a parallel beam of monoenergetic low energy electrons incident on the surface of a Ni crystal with Bragg planes spaced by $d=0.91 \AA$, 
obtained from previous X-ray scattering experiments on crystalline nickel [13]. In this experiment the applied accelerating voltage of the electrons beam was 54 volts, and the de Broglie wavelength obtained via Bragg's law was $\lambda=1.65 \AA$. According to the EFWP predictions, Equation (23), the de Broglie wavelength measured in the graphic is $\lambda=1.66 \AA$, what is in very good agreement with the experimental result. See Figure 4.

The results displayed in Figure 3 and Figure 4 show strong evidences that the electric field wave packet associated to an electron is the agent responsible for the waving aspect associated to the electron.

\section{The EFWP and Young's Double-Slit Experiment}

Armed with the ideas and the informations obtained in the previous sections, we are now going to discuss the applicability of the EFWP, in a double-slit experiment, for explaining the behavior and results predicted by this new electric field wave packet, taking a very qualitative procedure to face the problem. For doing this, let us consider a source $F$ emitting a narrow beam of electrons striking on an opaque plate $S$, where two narrow slits $S_{1}$ and $S_{2}$ were made. A screen $P$, a photographic film for example, is appropriately placed in front the plate $S$. This arrangement is called Young's double-slit experiment and it is described and illustrated with details in many quantum mechanics text books [1] [2] [11] and, we are not reproducing it here. Without entering into details of the experimental arrangement, we firstly consider a situation for which the slit $S_{2}$ is blocked and $S_{1}$ is open. In a situation like this we will obtain on the screen $P$ a distribution of fringes of the electric field $E_{1}(x)$ with an intensity $I_{1}(x)=\left|E_{1}(x)\right|^{2}$, representing the diffraction pattern of electrons passing through $S_{1}$. For example, using the Equation (23), an electron accelerated through a potential of $100 \mathrm{~V}$, passing through the slit $S_{1}$, will present on the screen $P$, the intensity $I_{1}(x)$ represented by the expression

$$
I_{1}(x)=\left|B^{2}\left(\frac{\cos ^{2}(1.88 x)}{x^{2}}+\frac{1.88}{x} \sin (2 \times 1.88 x)\right)\right|^{2}
$$

where $B=14.40 \times 10^{-10} \mathrm{~N} / \mathrm{C}$. The diffraction pattern, as predicted by Equation (27) is shown in Figure 5, where we can observe that, due to the $1 / x^{2}$ dependence of the electric field, the central peak of the diffraction pattern, at the position where the electron is more likely to strike the screen, $x=0$, is much more intense than the lateral peaks. The intensity of the electric field, as predicted by Equation (27), is mathematically divergent at the position of the particle, what is a characteristic of the potential used. But, from a physical point of view,

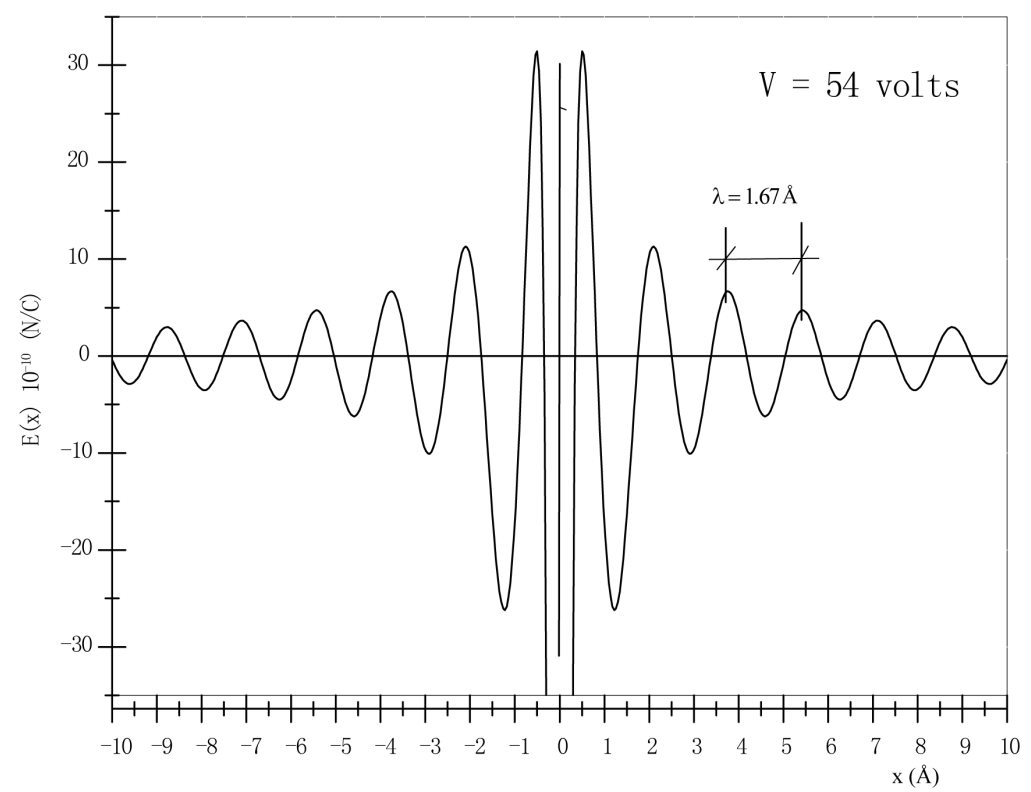

Figure 4. The electric field $E(x)$ for an unbound electron that has been accelerated through $V=54$ volts. The de Broglie wavelength measured in the graphic is $\lambda=1.66$ A. For Ni crystal, the Davisson and Germer experimental value is $\lambda=1.65 \AA$. 


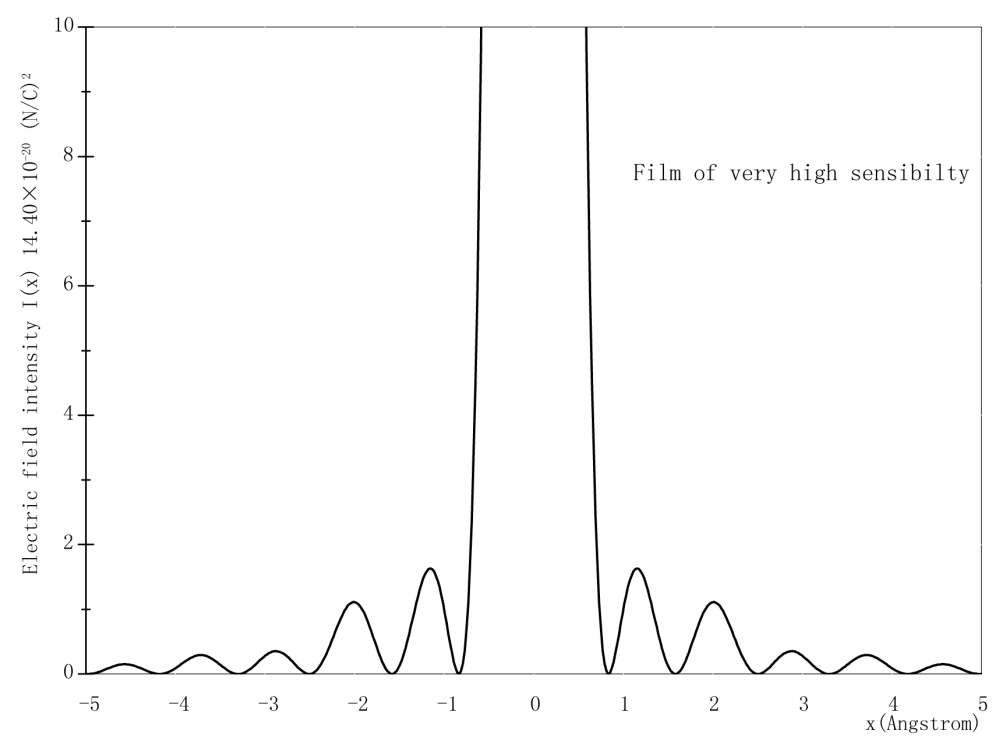

Figure 5. A diffraction pattern produced by one electron accelerated through 100 volts striking on the screen $\mathrm{P}$ of the double-slit apparatus,considering just one slit open and a film with high sensibility. Due to the $1 / r^{2}$ dependence of the electric field wave packet, the central peak of the diffraction pattern is much more intense than the lateral peaks.

because the electron has a very small but a finite size, the intensity $I(x)$, as $x \rightarrow 0$, can be very large indeed but having a finite value as have been shown in the end part of Section 2. The heights of the lateral peaks decrease as modulated by the classical Coulomb electric field. As we will see below, this characteristic of the electric field, will allow us to interpret the particle-wave duality behavior of the electron, using a quite different point of view. A similar diffraction pattern, $I_{2}(x)=\left|E_{2}(x)\right|^{2}$, of electric field $E_{2}(x)$, will be obtained if $S_{1}$ is blocked and $S_{2}$ is open. Now, if we consider that the number of electrons per unit of time, passing through the two slits, not simultaneously open, is the same, the two diffraction patterns become equals and they will appear as displayed in Figure 5, but with an intensity that will depend on the number of electrons per unit of time are striking the film surface. However, when the two slits $S_{1}$ and $S_{2}$ are open, simultaneously, and considering a difference of phase $\phi_{1}$ and $\phi_{2}$ between the fields $E_{1}(x)$ and $E_{2}(x)$, what we will observe on the screen $P$ is a pattern of interference of fringes $E(x)=E_{1}(x)+E_{2}(x)$, which will not be the sum of the intensities $I_{1}(x)$ and $I_{2}(x)$ as obtained separately. Instead, the intensity $I(x)$ of the two fields is represented by the expression [5] [12]

$$
I_{x}(x)=\left|E_{1}(x)+E_{2}(x)\right|^{2}=\left|E_{1}(x)\right|^{2}+\left|E_{2}(x)\right|^{2}+2\left|E_{1}(x)\right|\left|E_{2}(x)\right| \cos \left(\phi_{2}-\phi_{1}\right) .
$$

The term $2\left|E_{1}(x)\right|\left|E_{2}(x)\right| \cos \left(\phi_{2}-\phi_{1}\right)$ in the Equation (28) represents the interference of the two fields $E_{1}(x)$ and $E_{2}(x)$.

Now, let us consider a situation in which the source $F$ emits electrons, practically one by one. With this condition, three distinct situations may occur:

1) Let us assume that only one of the slits, lets say, $S_{1}$ is open. Considering that the sensibility of the film is very... very high, according to Equation (25), what we must observe on the screen $P$ is a diffraction pattern of one electron, with very... very small but not vanishing intensity of the lateral peaks. In this case, we would say that the electron presents the character of wave. This situation is shown in Figure 5. By other side, increasing the number of electrons of the beam per unit of time, or equivalently, increasing the time of exposure of the film, the diffraction pattern will become more, and more accentuated, without changing its waving aspect for the production a diffraction pattern. This condition is shown in Figure 6, where it was considered that 1, 5 and 50 electrons per unit of time were striking on the surface of the film of very... very high sensibility. Increasing the number of electrons in the beam, we can see that the diffraction pattern will be preserved.

2) Let us take another consideration, if the sensibility of the film is not necessarily very high, what we will 


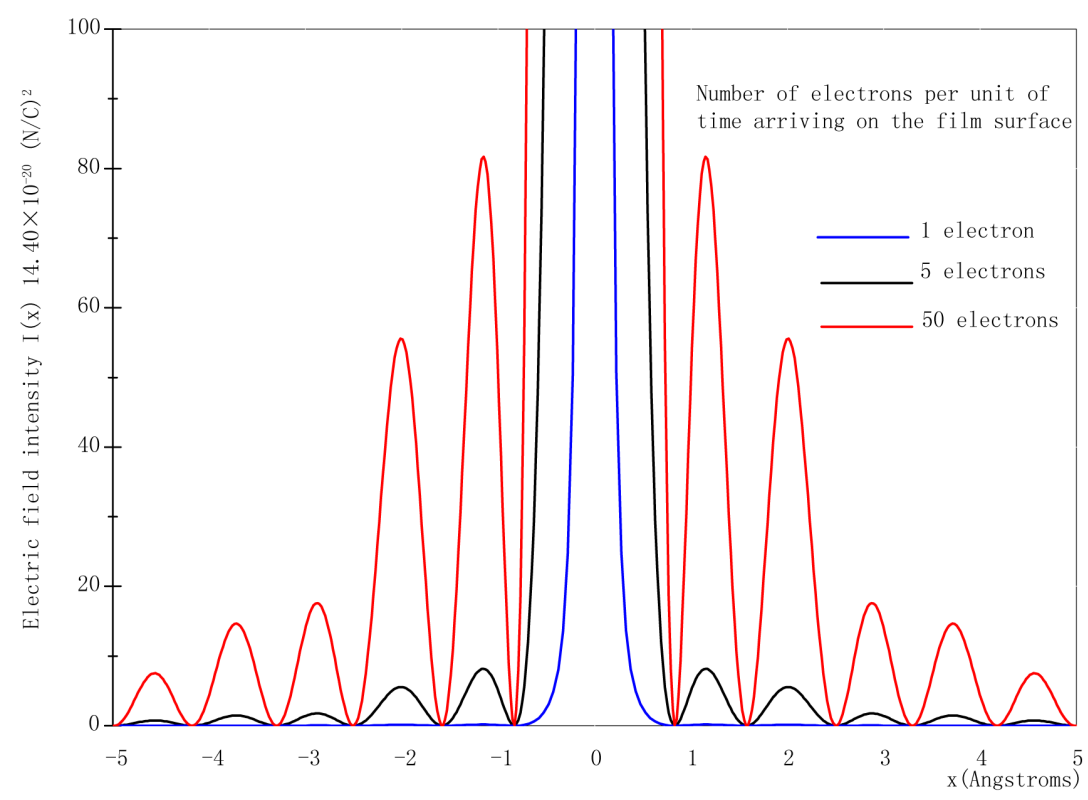

Figure 6. The diffraction pattern of beam of 1,5 and 50 electrons per unit of time passing through the double-slit experiment, with just one slit open. It is observed that the intensity of the lateral peaks of the electric field is quite sensible to the number of electrons in the beam.

expect to observe on the screen $P$ is only the signal produced by the central peak of the diffraction pattern for single electron, which due to the character of the electric field it is of the order of $10^{13}$ more intense than the lateral peaks. In a situation like this, what we expect to see on the screen $P$ is a small spot, similar to the mark produced by a single particle striking on the film surface. A simulation of this situation is displayed in Figure 7, where the intensities of the lateral peaks is considered too small to be observed. In a situation like this, we would say that the electron presents the character of a particle.

3) For last, let us consider a probabilistic interpretation for observing the interference phenomenon of electrons in the double-slit experiment. As we can see in Figure 3, the effective size of the electric wave packet around the electron is about $10 \AA$. Thus, if we consider the aperture of the slits $S_{1}$ and $S_{2}$ is anything larger than this value, let's say $15 \AA$ and the slits $S_{1}$ and $S_{2}$ open, what we will expect to observe on the film is a certain number of electrons of the two beams colliding with the edges of the slits. These electrons will be scattered randomly within a certain angle $\theta$ before reaching the plane of the film. The remainder electrons of the beams will pass through the slits $S_{1}$ and $S_{2}$ without being scattered by the borders. Now, let us consider that the number of electrons in the beams is very small, passing one by one through the slits. Then, according to the situation number 2, described above, what we expect to see is a random distribution of small spots on the film surface, producing a pattern of at least three strips of spots. Two of them, with larger concentration produced by electrons not scattered by the edges of the slits. A third one appearing between these two, strips, produced by cross scattering of electrons, occurring on the upper and the lower edges of the two slits. Furthermore, considering a small number of electrons in the beam, what must be observed in this case is a random distribution of spots on the film, but preserving the behavior of particles in the pattern. See Ref. [14]. Otherwise, increasing considerably the number of the electrons in the beam, the lateral peaks of individual electron diffraction pattern begin to increase in certain regions of the film, due to constructive interference of the electric field and decreasing in other regions due to destructive interference, in such a way that a complete interference pattern begins to be formed on the film surface. In a situation like this, we would say that the electrons behave as wave.

Another interesting conclusion we can draw from this interpretation is the controversial discussion about the change of character of the particle, be it a particle or be it a wave, can be affected by the intervention of the observer in the experiment. According to the interpretation presented above, our answer is no. To give the negative answer to this question, will consider two different situations:

1a) Let's assume that the number of electrons passing through either slit is not very high, in such a way that 


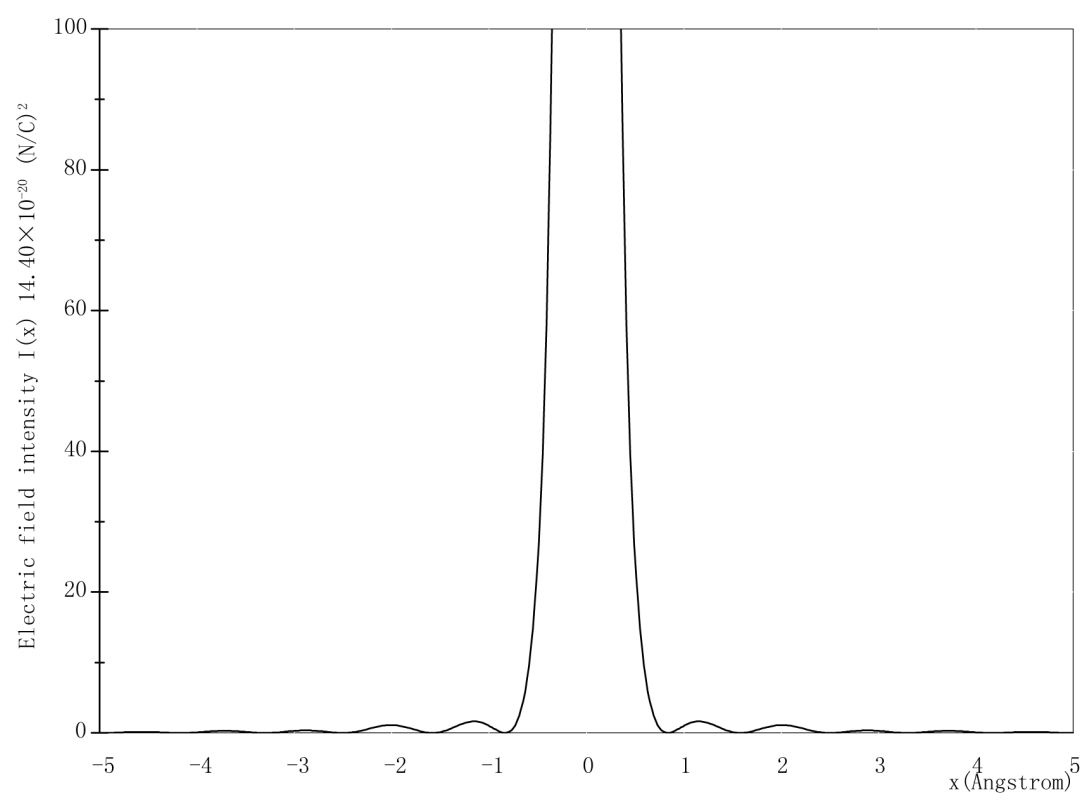

Figure 7. Simulation of the diffraction pattern of an electron, striking on a film of very low sensibility. Only the central peak of the pattern is appearing as a spot on the film. The spot is the mark left by the electron behaving as a particle.

we can count them using a detector. The fact of placing a detector near one of the slit, to see if the electron went through that particular slit, will not affect the electrons that are going through the other slit. Thus, even considering that the detector placed next to one of the slit can block it, preventing the electrons from reaching the phosphorescent screen, will not invalidate the experiment and will not change the character of the particle. Because, as the number of electrons in the beam have been considered small, the intensity of electric field on the screen also will be small. Thus, the randomness of the scattering of electrons at the edges of the slit not blocked, will cause only a random distribution of small spots on the screen, produced by the large central peak of the electric field intensity. Thus, as explained previously, the electrons will be observed as particles.

1b) Now, let us consider situation of (1a) but with the number of electrons in the beam being very... very large. In this case, what we expect to observe on the screen is diffraction pattern produced by the superposition of the random distribution of central and lateral peaks, interfering constructively or destructively, to form the diffraction pattern. In a situation like this we would say that the electrons behave as waves. Thus, the presence of a detector near one of the slits is will not affect the results of the experience as predicted by other interpretations of the phenomenon.

2) Now let us consider that both slits are open and without the presence of the detector. Let us also assume that the number of electrons in the beam is large, or equivalently, the number of electrons can be considered small but the exposure time is large. The result will be the same. The constructive or destructive interference of central and side peaks of electric field intensity in the phosphorescent screen, will produce an interference pattern. In this case, our conclusion is that the electrons will behave like a waves. Thus, according to this new model, what are the facts more important to be observed in the experiment, to conclude whether the electron is particle or wave. First, the number of electrons in the beam or the time of exposure of the film. Second, the sensibility of the film is also important.

In Section 1, we called the reader's attention to the possible existence of a bosonic atom in a dense beam of electron, similar to the existence of electron Cooper pairs for some metals in the state of superconductivity. This new atom could be seen as a particle with twice the mass of the electron, $m_{b a}=2 m_{e}$ and having twice its electric charge, $q_{b a}=2 e$. As seen in Figure 2, the ground state of this new atom is formed of two electrons separated from each other by an average distance $\langle d\rangle=1.06 \AA$. At this distance, the electric force between them is null and, if they are in a state of spin $S=0$ (unpaired spins), what exist between two electrons is an attractive magnetic force, given to the system a certain degree of stability, forming what we are calling bosonic atoms produced by the interaction of two electrons. Thus, if we assume that in a double-slit experiment, with the 
use of a dense beam of particles, electrons and bosonic atoms and the beam is subjected to a given accelerating potential of $V_{0}$, what kind of diffraction pattern will be observed in the phosphorescent screen of the device? In Equation (15) we can observe that due to its larger mass the bosonic atom, twice times larger, should be twice times slower than the isolated electron. At the same time, it should be twice times faster due to its larger charge, twice times larger. So, what the bosonic atom loses by one side is compensate by the other. Being so, the time that both particles take from leaving the source and reaching the end of the trip is the same because they have the same velocity. See Equation (24) to make sure of this statement.

Finally, let us consider what kind of change may occur in the diffraction pattern of electrons admitting the presence of bosonic atoms in the electron beam. Figure 2 shows us that in the ground and the first excited states of this new kind of atoms the two electrons in the atom are separated from each other, respectively, by an average distance of $1.06 \AA$ and $4.24 \AA$. Thus, for a given number of electrons arriving on the film surface, what we expect to see in the diffraction pattern is certain number of isolated spots produced by isolated electrons colliding with the film and other number of pair of spots randomly distributed with regular distances, compatible with the separation of the two electrons in the existing pairs in the beam of electrons. To our surprise and delight, an experiment to illustrate our forecast has been carried out by E. R. Huggins, Physics I. The diffraction pattern in a double slit experiment is reproduced in the book-Modern Physics-P. A. Tipler and R. A. Llewellyn, W. H. Freeman and Company, N. Y. 2014 [14].

The experiment conducted by Huggins, clearly shows the evolution of the mechanism for the passage from a random scattering of particles (random formation of spots on the photographic film) to the formation of beautiful interference pattern, when the number of electrons in the beam is greatly increased. By carefully observing the evolution of the figures obtained in the experiment, we can clearly see the existence of pairs of spots, randomly distributed on the film. Does it constitute a proof for the existence of the bosonic atoms in a dense beam of electrons?

This simple qualitative interpretation presented above, was strongly suggested or induced by the results experimentally observed with electrons scattering in many crystalline solids. In a situation like this, the figure observed on the plane of the film must be concentric bright and dark alternating rings of decreasing intensities from the center to the border of the pattern. Due to the $1 / r^{2}$ dependence of the electric field wave packet, the central peak of the diffraction pattern must be more bright than the others. In fact, this is the pattern experimentally observed with electrons diffraction in many cryslalline solids.

The explanation given above, even being considered very simple, it constitutes a different point of view for a probabilistic interpretation of the phenomenon of diffraction or interference produced by electrons in solids. According to this interpretation, the acceptance of the electron as a particle or as wave will depend on how the experiment is performed. Another aspect that is also clear is that the intervention of the observer, is not a determining factor to say whether the electron is a particle or a wave. This is an irrelevant condition to the process of observation. What is clear with this interpretation is that, even coexisting at the same time, both characters, wave or particle, cannot be observed simultaneously. This is a fact known since long time ago. What is new in this presentation is the use of an electric field wave packet, which enabled us to present a new and a quite different interpretation for the phenomenon of the particle-wave duality and, we believe, more palatable model than others, formulated previously, to achieve this purpose.

\section{Conclusion and Remarks}

In this work, we have used the Quantum Oscillatory Modulated Potential to investigate the phenomenon of wave particle duality. With this new potential, we have shown that the electric field existing in the vicinity of a microscopic electric particle in movement, has the form of a wave packet surrounding the particle. The new electric field wave packet allowed us to present a new interpretation for the double-slit experiment with a beam of electrons, in a much more simple way. We have shown that the magnitude of the de Broglie wavelength associated to this electric field wave packet is dependent on the mass of the particle and its charge. As it is well known since long ago the wavelength, decreases when the mass of particle increases. However, according to the arguments and calculations presented in this work, the origin and the waving behavior of the particle are being ascribed to the electric field produced by the charge and not to the mass of the particle. According to this model and based on experimental results, a new interpretation was presented to explain the diffraction pattern of electrons in a double slit experiment. Besides its simplicity, the results predicted by the new model of electric 
field wave packet are in very good agreement with the experimental data, obtained with diffraction of electrons in many crystalline solids. Furthermore, as an additional information, using a point of view which can be considered rather audacious, the work points out indications for the existence of a kind of bosonic atoms in a narrow and dense beam of electrons as well as in a sea of electrons near the Fermi surface in superconducting metals at temperatures below $T_{c}$, forming what is called Cooper pairs of electrons. The observed Cooper pairs in Josephson effects with supercurrent tunneling also may be an indicaton for the existence of the new kind of bosonic atom formed by the interaction of two electrons.

\section{References}

[1] Greene, B.R. (2014) The Elegant Universe: Superstring and Hidden Dimensions. Title in Portuguese: O Universo Elegante. Translated by José Viegas Filho, Editora Schwarcz S.A.

[2] Feymann, R.P., Leighton, R.B. and Sands, M. (1966) Lectures on Physics. Addison Wesley, Reading.

[3] McQuarrie, D.A. (1983) Quantum Chemistry. University Science Books.

[4] Eisberg, R.M. (1961) Fundamentals of Modern Physics. John Wiley \& Sons, Inc., New York.

[5] Wolney Filho, W. (2014) Mecânica Quântica. 2nd Edition, Editora da Universidade Federal de Goiás, Goiânia.

[6] Wolney Filho, W. (2012) Journal of Modern Physics, 3, 660-664. http://dx.doi.org/10.4236/jmp.2012.38090

[7] Ohannian, H.C. (1985) Physics. Norton \& Company, Inc., N.Y.

[8] Altarev, I.S., et al. (1980) Nuclear Physics A, 341, 269. http://dx.doi.org/10.1016/0375-9474(80)90313-9

[9] Pospelov, M. (2005) Annals of Physics, 318, 119. http://dx.doi.org/10.1016/j.aop.2005.04.002

[10] Kittel, C. (1986) Introduction to Solid State Physics. John Willey \& Sons, New York.

[11] Bardeen, J., Cooper, L.M. and Sherieffer, J.R. (1957) Physical Review, 5, 1175. http://dx.doi.org/10.1103/PhysRev.108.1175

[12] Cohen-Tannoudji, C., Diu, B. and Laloe, F. (1977) Quantum Mechanics. Vol. 1, John Willey \& Sons, New York.

[13] Johnsons, T.H. (1931) Physical Review, 37, 847. http://dx.doi.org/10.1103/PhysRev.37.847

[14] Tipler, P.A. and Llewellyn, R.A. (2014) Fsica Moderna, sexta edição, Tradução: Ronaldo S. Biasi, LTC, Rio de Janeiro. 